\title{
General considerations on lepton mass matrices
}

\author{
Yakefu Reyimuaji \\ SISSA/ISAS and INFN, Via Bonomea 265, 34136 Trieste, Italy \\ ICTP, Strada Costiera 11, 34151 Trieste, Italy \\ E-mail: yreyimua@sissa.it

\section{Andrea Romanino*} \\ SISSA/ISAS and INFN, Via Bonomea 265, 34136 Trieste, Italy \\ ICTP, Strada Costiera 11, 34151 Trieste, Italy \\ E-mail: romaninodsissa.it
}

\begin{abstract}
We classify the flavour groups and representations providing, in the symmetric limit, an approximate description of lepton masses and mixings. We assume that the light neutrinos are of Majorana type and that the flavour symmetry directly constrains their mass matrix. The representations can be characterised by the dimension, type (real, pseudoreal, complex), and equivalence of its irreducible components, and in terms of such a classification we find only six viable cases. It turns out that the neutrinos are always either anarchical or have an inverted hierarchical spectrum. Therefore, if the hint of a normal hierarchical spectrum were confirmed, we would conclude (under the above assumption) that symmetry breaking effects must play a primary role in the understanding of neutrino flavour observables.
\end{abstract}

Corfu Summer Institute 2017 'School and Workshops on Elementary Particle Physics and Gravity' 2-28 September 2017

Corfu, Greece

${ }^{*}$ Speaker. 


\section{Introduction and definition of the problem}

Symmetries are among the tools that proved most successful in the understanding of the patterns of physical phenomena. It is not unconceivable that they also play a role in the understanding of the pattern of fermion masses and mixings in the Standard Model (SM) (here meant to include the necessary ingredients needed to account for neutrino masses).

Indeed, flavour symmetries [1-15] have long represented one of the most popular attempt in this direction. Over the years, and especially after data on lepton mixing become available, shifting the attention from quark to lepton masses and mixings, the role of flavour symmetries has quite changed. Initially, the symmetry was supposed to provide an approximate understanding of the pattern of masses and mixing in the unbroken limit. On the other hand, lately more and more often, the symmetry itself does not provide an insight on lepton mixing, as the mixing ends up being arbitrary in the symmetric limit; it is the pattern of symmetry breaking that really shapes the flavour pattern. The question we want to address is then: are lepton masses and mixings determined by a symmetry or by its breaking?

In order to provide a precise formulation of the problem, we consider a generic (continuous or discrete, simple or not, abelian or non-abelian, or arbitrary combinations of the above) flavour group $G$ broken to a subgroup $H$. We write the charged lepton and neutrino mass matrices $m_{E}$ and $m_{v}$ as

$$
\begin{aligned}
& m_{E}=m_{E}^{(0)}+m_{E}^{(1)} \\
& m_{v}=m_{v}^{(0)}+m_{v}^{(1)},
\end{aligned}
$$

where $m_{E}^{(0)}, m_{v}^{(0)}$ are invariant under $G$, while $m_{E}^{(1)}, m_{v}^{(1)}$ are generated by the sources of symmetry breaking, and vanish in the symmetric limit. The non-vanishing entries in $m_{E}^{(0)}, m_{v}^{(0)}$ are assumed to be of the same order, according to the principle that flavour hierarchies should be accounted for by the flavour model itself. The size of the corrections associated to the symmetry breaking effects is assumed to be smaller.

We aim at classifying all flavour symmetry groups and representations such that $m_{E}^{(0)}, m_{v}^{(0)}$ provide an approximate description of lepton flavour observables, while $m_{E}^{(1)}, m_{v}^{(1)}$ only provide the moderate correction necessary for an accurate description. Given the large variety of possible cases, it is not a priori obvious that a complete analysis can be carried out in an effective way and would produce results that can be expressed in a concise form. Interestingly, this turns out to be the case: the problem can be studied in full generality, admits a precise mathematical formulation, and a complete and compact solution, in the assumptions that i) the light neutrino masses are in Majorana form and ii) the symmetry arguments can be applied directly to the light neutrino mass matrix (or to the Weinberg operator from which it originates).

We say that $m_{E}^{(0)}, m_{v}^{(0)}$ provide an approximate description of lepton flavour observables if:

- The charged lepton and neutrino masses obtained from their diagonalisation are in the form shown in table 1, so that only a small correction is required in order to provide an accurate description of the lepton spectrum.

- All the entries of the PMNS matrix obtained from $m_{E}^{(0)}, m_{v}^{(0)}$ are non-vanishing, except per- 


\begin{tabular}{|c|c|c|}
\hline & $\begin{array}{l}\text { non-zero entries } \\
\text { of the same order }\end{array}$ & $\begin{array}{l}\text { hierarchy among } \\
\text { non-zero entries }\end{array}$ \\
\hline $\begin{array}{l}\text { charged } \\
\text { leptons }\end{array}$ & $(A, 0,0)$ & $\begin{array}{l}(A, B, 0) \\
(A, B, C)\end{array}$ \\
\hline $\begin{array}{c}\text { neutrinos } \\
\mathrm{NH}\end{array}$ & $(a, 0,0)$ & \\
\hline neutrinos & $(a, a, a)$ & $(a, b, 0)$ \\
\hline $\mathrm{NH}$ or $\mathrm{IH}$ & $(a, b, b)$ & $(a, b, c)$ \\
\hline $\begin{array}{c}\text { neutrinos } \\
\text { IH }\end{array}$ & $(0, a, a)$ & \\
\hline
\end{tabular}

Table 1: Charged lepton and neutrino mass patterns in the symmetric limit.

haps the 13 element ${ }^{1}$. As all of the PMNS entries appear to be of order one, with the exception of the 13 element, only a small correction is then required in order to provide an accurate description of the PMNS matrix.

In contrast, the lepton mixing (and a part of the mass spectrum) are fully determined by symmetry breaking when, for example, all neutrino or all charged lepton masses identically vanish in the symmetric limit.

In the first column in table 1 we list the cases that can be considered as good leading order approximations even when all the non-zero entries are of the same order of magnitude. The cases in the second column, on the contrary, require some degree of hierarchy or degeneracy between the non-zero entries. Such a distinction is more important for charged leptons than neutrinos: in the neutrino case only mild hierarchies up to $\mathscr{O}(5)$ are required to account for $\Delta m_{12}^{2} /\left|\Delta m_{23}^{2}\right| \ll 1$ (in the normal hierarchy case). Therefore, we will only care about the distinction between first and second column in the case of charged leptons. In the case of neutrinos, we distinguish the cases leading (after taking into account small symmetry breaking corrections) to a normal hierarchy (NH), an inverted hierarchy $(\mathrm{IH})$, or to any of the two depending on the sizes of the non-zero entries. A normal hierarchical spectrum is at present favoured by data [16-18], but we still retain the inverted spectrum as a viable possibility.

\section{Analysis}

Having specified the mass and mixing patterns that we consider viable in the symmetric limit, we now want to characterise the flavour groups and representations leading to any of those patterns. Let us then give first of all a precise formulation of the problem.

The flavour symmetry group $G$ acts on the SM Weyl leptons $l_{i}$ and $e_{i}^{c}$ through unitary representations $U_{l}$ and $U_{e^{c}}$ respectively. Here $e^{c}$ is the $\mathrm{SU}(2)_{L}$ singlet with hypercharge $Y=1$. With this notation, all the fermion fields are left-handed. The charged lepton and neutrino mass matrices

\footnotetext{
${ }^{1}$ For at least one choice of the mass eigenstates. Such specification is necessary in the cases in which some masses are degenerate in the symmetric limit.
} 
arise from the Yukawa and Weinberg operators [19] respectively,

$$
\lambda_{i j}^{E} e_{i}^{c} l_{j} h^{*}, \quad \frac{c_{i j}}{2 \Lambda} l_{i} l_{j} h h,
$$

and are given by

$$
m_{E}=\lambda_{E} v, \quad m_{v}=c v^{2} / \Lambda,
$$

where $h$ is the Higgs field, $v=|\langle h\rangle|$, and Lorentz-invariant contractions of fermion indices are understood.

The symmetric part of the mass matrices $m_{E}^{(0)}, m_{v}^{(0)}$ satisfy

$$
m_{E}^{(0)}=U_{e^{c}}^{T}(g) m_{E}^{(0)} U_{l}(g) \quad m_{v}^{(0)}=U_{l}^{T}(g) m_{v}^{(0)} U_{l}(g) \quad \forall g \in G .
$$

A possible non-trivial transformation of $h$ under $G$ can be reabsorbed in $U_{l}$ and $U_{e^{c}}$.

We want to determine, or characterise, all groups $G$ and representations $U_{l}, U_{e^{c}}$ such that any $m_{E}^{(0)}, m_{v}^{(0)}$ satisfying eq. (2.3) provide an approximate description of lepton flavour, as defined above. We also require of course the existence of at least one example of such $m_{E}^{(0)}, m_{v}^{(0)}$ (with all masses that are allowed to be different and non-zero indeed different and non-zero).

We now illustrate the results of the analysis. The details and full proofs can be found in [20]. First of all, it turns out that the pattern of lepton masses and mixings only depends on the dimension, type (real, pseudoreal, complex), and equivalence of the irreducible components of the representations $U_{l}, U_{e^{c}}$. We then classify the groups and representations leading to a viable symmetric limit in terms of the structure of the decomposition of $U_{l}, U_{e^{c}}$ into irreducible representations (irreps). We find only six viable cases, shown in table 2 .

Each pattern may correspond to different flavour groups and representations, provided that the decomposition of the representation on the leptons follows that pattern. The allowed patterns contain one-dimensional irreps only. Pseudoreal representations do not play a role.

Three out of the six cases in the table are partially trivial. Those are the cases in which $U_{l} \sim$ $1+1+1$, for which the representation on the lepton doublets is either the identity representation or an overall sign change. In such a case, the neutrino mass matrix is not constrained at all, and the neutrino masses and PMNS matrix are expected to be completely generic. In particular, the relative smallness of $\left|(U)_{13}\right|$ is accidental. We are in the presence of "anarchical" neutrinos [21,22]. The only constraints that can be obtained are on the charged lepton masses, through the interplay of the trivial $U_{l}$ with a non-trivial $U_{e^{c}}$.

The other three cases provide non-trivial constraints on neutrino masses and mixings. An important result is that they all correspond to inverted neutrino hierarchy, and specifically to two degenerate and one vanishing neutrino mass in the symmetric limit. Therefore, if the present hint favouring a normal hierarchy were confirmed, we would conclude, within our assumptions, that either the flavour model is not predictive at all in the neutrino sector, or the symmetric limit does not provide an approximate description of lepton masses and mixings.

Table 2 is divided in two parts. In the first part, the hierarchy of the charged lepton masses is naturally accommodated by the vanishing of the two lighter masses in the symmetric limit, in agreement with the principle that hierarchies should be explained by the flavour model. In the second part, hierarchies not accounted for by the flavour theory have to be invoked among the nonzero entries in order to account for the structure of charged lepton masses. The second case in the 


\begin{tabular}{|c|c|c|c|c|c|}
\hline irreps & masses & $v$ hierarchy & $\begin{array}{llllll}H_{E} & P_{E} & V & D & P_{V} & H_{V}\end{array}$ & $U$ & zeros \\
\hline $\begin{array}{lll}1 & 1 & 1 \\
1 & r & \nsupseteq 1\end{array}$ & $\begin{array}{l}(A 00) \\
(a b c)\end{array}$ & $\mathrm{NH}$ or $\mathrm{IH}$ & $V$ & $V$ & none \\
\hline $\begin{array}{l}\mathbf{1} \mathbf{1} \overline{\mathbf{1}} \\
\overline{\mathbf{1}} r \nsupseteq \mathbf{1}, \overline{\mathbf{1}}\end{array}$ & $\begin{array}{l}(A 00) \\
(0 a a)\end{array}$ & $\mathrm{IH}$ & $V_{23} D_{12}$ & $H_{12}^{E} V_{23} D_{12}^{-1}$ & none $(\mathbf{1 3})$ \\
\hline $\begin{array}{lll}1 & 1 & 1 \\
1 & 1 & r \neq 1\end{array}$ & $\begin{array}{l}(A B 0) \\
(a b c)\end{array}$ & $\mathrm{NH}$ or IH & $V$ & $V$ & none \\
\hline $\begin{array}{l}\mathbf{1} \mathbf{1} \overline{\mathbf{1}} \\
\overline{\mathbf{1}} \overline{\mathbf{1}} r \neq \mathbf{1}\end{array}$ & $\begin{array}{l}(A B 0) \\
(0 a a)\end{array}$ & $\mathrm{IH}$ & $V_{23} D_{12}$ & $V_{23} D_{12}^{-1}$ & 13 \\
\hline $\begin{array}{lll}1 & 1 & 1 \\
1 & 1 & 1\end{array}$ & $\begin{array}{l}(A B C) \\
(a b c)\end{array}$ & $\mathrm{NH}$ or IH & $V$ & $V$ & none \\
\hline $\begin{array}{lll}1 & 1 & \overline{1} \\
\overline{1} & \overline{1} & 1\end{array}$ & $\begin{array}{l}(A B C) \\
(0 a a)\end{array}$ & $\mathrm{IH}$ & $P_{E} \quad V_{23} D_{12}$ & $P_{E} V_{23} D_{12}^{-1}$ & $\mathbf{1 3}, 23,33$ \\
\hline
\end{tabular}

Table 2: Irrep decompositions of $U_{l}, U_{e^{c}}$ giving rise, in the symmetric limit, to an approximate description of lepton flavour observables. The first column shows the decomposition of $U_{l}$ and $U_{e^{c}}$, one above the other. Only real and complex (no pseudoreal) irreps appear. Boldface fonts denote complex representations, regular fonts denote real representations. Primes are used to distinguish inequivalent representations, and in the case of complex representations $\mathbf{1}^{\prime}$ is supposed to be different from both $\mathbf{1}$ and $\overline{\mathbf{1}}$. " $r$ " denotes a generic, possibly reducible representation, different from or not including the specified irreps, as indicated. The second column shows the corresponding pattern of charged lepton and neutrino masses in the symmetric limit, one above the other, and the third the neutrino hierarchy type, normal $(\mathrm{NH})$ or inverted $(\mathrm{IH})$. The decomposition of the PMNS matrix according to eq. (2.4) then shown. A matrix with no further specification is generic (e.g. $P$ denotes a generic permutation, $V$ a generic unitary matrix). $D_{i j}$ denotes a $\pi / 4$ rotation in the generic form in eq. (2.5) acting in the sector $i j$. The presence and position of a zero in the PMNS matrix in the symmetric limit is specified in the last column.

first part of the table is special, as the size of the 13 element of the PMNS matrix is determined by the rotation $H_{12}^{E}$, which is not physical in the symmetric limit, and will be fixed by the symmetry breaking effects generating the muon mass. Depending on the structure of those effects, the size of $(U)_{13}$ can end up being large, small, or zero. Finally, note that since the parameters entering all the mixing matrices in table 2 except $D$ are generic, a specific value of a mixing angle can be obtained only when the matrix $D$ is involved. As the table shows, $D$ can only play a role in the 12 mixing, in agreement with earlier specific results [23].

The form of the PMNS matrix associated to each of the cases in table 2 can be obtained without the need of diagonalising, or even knowing, the explicit form of the lepton mass matrices, as it only depends on the structure of the irreps decomposition of $U_{l}$ and $U_{e^{c}}$. The PMNS matrix can in fact be written in the form

$$
U=H_{E} P_{E} V D^{-1} P_{v}^{-1} H_{v}^{-1} .
$$

The contributions to $U$ on the right hand side have different origins and different physical meanings. Each of them can be obtained as follows.

- $V$ is a generic unitary transformation commuting with $U_{l}$, with $\mathscr{O}(1)$ entries. Its origin is associated to the presence of equivalent copies of the same irrep type in the decomposition 
of $U_{l}$. If all the irrep components are inequivalent, $V$ is trivial.

- $D$ is associated to the possible presence of a Dirac sub-structure in the neutrino mass matrix, and it originates from the presence of complex conjugated irreps within the decomposition of $U_{l}$. In the three neutrino case, there are only two possibilities. Either $U_{l}$ does not contain pairs of complex conjugated irreps, in which case $D$ is trivial, $D_{i j}=\delta_{i j}$. Or there is one pair of one-dimensional complex conjugated representations, in the positions $i$ and $j$ in the list of irreps, in which case $D$ is a maximal $2 \times 2$ rotation,

$$
D_{2}=\frac{1}{\sqrt{2}}\left(\begin{array}{cc}
1 & 1 \\
-i & i
\end{array}\right),
$$

embedded in the $i j$ block. The corresponding mass eigenvalues are degenerate.

- The permutation matrices $P_{E}$ and $P_{V}$ are associated to the possible need of reordering the list of eigenvalues. Indeed, the list of eigenvalues obtained with the above rules is not necessarily in the standard ordering, required for a proper definition of the PMNS matrix.

- Finally, the role of $H_{e}, H_{v}$ is to take into account possible ambiguities in the definition of the PMNS matrix in the symmetric limit. In the real world, all leptons are non-degenerate and the PMNS matrix only has unphysical phase ambiguities, which do not need to be taken into account. When considering the symmetric limit, on the other hand, larger ambiguities can arise due to degenerate, possibly vanishing, masses. In practice, $H_{E}$ is a generic unitary transformation mixing the massless charged leptons; and $H_{v}$ contains a generic unitary transformation mixing the massless neutrinos and a generic orthogonal transformation mixing degenerate massive neutrinos (it turns out, however, that the latter can be ignored if the degeneracy is due to a Dirac structure, in which case it can be reabsorbed into a phase redefinition of $V$ ). The $H_{e}, H_{v}$ contributions to the PMNS matrix have a different physical nature than the previous ones. They are unphysical, and undetermined, in the symmetric limit. However, they become physical (up to diagonal phases) after symmetry breaking effects split the degenerate mass eigenstates. Depending on the specific form of the symmetry breaking effects, $H_{e}$ and $H_{v}$ can end up being large, small, or zero (unlike the previous contributions, which are determined by the non-zero entries and are large in the absence of accidental correlations [24]), with possible consequences on the size of $U_{13}$ [25-36].

Further details and examples can be found in [20].

\section{Summary}

In summary, we provided a complete answer to the following question: what are the flavour groups, of any type, and representations providing, in the symmetric limit, an approximate description of lepton (fermion) masses and mixings? Despite the generality of the problem, the complete answer is simple and has an important corollary: either the flavour symmetry does not constrain at all the neutrino mass matrix (anarchy), or the neutrinos have an inverted hierarchical spectrum. Therefore, if the present hint of a normal hierarchical spectrum were confirmed, we would conclude that, under the above assumption, flavour models leading to an approximate description of 
lepton masses and mixings in the symmetric limit are not able to account for any of the neutrino flavour observables, and symmetry breaking effects must play a primary role in their understanding. Such a conclusion is further strengthened in the case in which the representation of the flavour group commutes with the standard representation of a SU(5) grand unified gauge group. In the latter case, not even the options leading to an inverted hierarchical spectrum are available, and the only option is anarchy. In the case of $\mathrm{SO}(10)$, there are no solutions.

The main caveat to the previous conclusion is the assumption that the light neutrinos are of Majorana type, and that the symmetry arguments can be applied directly to their mass matrix. The origin of Majorana neutrino masses most likely resides at high scales, where additional relevant degrees of freedom (singlet neutrinos for example) might live. In such a case, the flavour symmetry acts on the high-scale degrees of freedom as well. The low-energy analysis turns out to be often equivalent to the high-scale analysis, but not always. Such a caveat will be studied in future work.

\section{References}

[1] C. D. Froggatt and H. B. Nielsen, Hierarchy of Quark Masses, Cabibbo Angles and CP Violation, Nucl. Phys. B147 (1979) 277-298.

[2] J. Bijnens and C. Wetterich, Fermion Masses From Symmetry, Nucl. Phys. B283 (1987) 237-267.

[3] M. Leurer, Y. Nir and N. Seiberg, Mass matrix models, Nucl. Phys. B398 (1993) 319-342, [hep-ph/9212278].

[4] M. Dine, R. G. Leigh and A. Kagan, Flavor symmetries and the problem of squark degeneracy, Phys. Rev. D48 (1993) 4269-4274, [hep-ph/9304299].

[5] L. E. Ibanez and G. G. Ross, Fermion masses and mixing angles from gauge symmetries, Phys. Lett. B332 (1994) 100-110, [hep-ph/9403338].

[6] A. Pomarol and D. Tommasini, Horizontal symmetries for the supersymmetric flavor problem, Nucl. Phys. B466 (1996) 3-24, [hep-ph/9507462].

[7] R. Barbieri, G. R. Dvali and L. J. Hall, Predictions from a U(2) flavor symmetry in supersymmetric theories, Phys. Lett. B377 (1996) 76-82, [hep-ph/9512388].

[8] C. D. Carone, L. J. Hall and H. Murayama, $\left(S_{3}\right)^{3}$ flavor symmetry and $p \rightarrow K^{0} e^{+}$, Phys. Rev. D53 (1996) 6282-6291, [hep-ph/9512399].

[9] E. Dudas, C. Grojean, S. Pokorski and C. A. Savoy, Abelian flavor symmetries in supersymmetric models, Nucl. Phys. B481 (1996) 85-108, [hep-ph/9606383].

[10] R. Barbieri, L. J. Hall, S. Raby and A. Romanino, Unified theories with U(2) flavor symmetry, Nucl. Phys. B493 (1997) 3-26, [hep-ph/9610449].

[11] R. Barbieri, L. J. Hall and A. Romanino, Consequences of a U(2) flavor symmetry, Phys. Lett. B401 (1997) 47-53, [hep-ph/9702315].

[12] C. D. Carone and L. J. Hall, Neutrino physics from a U(2) flavor symmetry, Phys. Rev. D56 (1997) 4198-4206, [hep-ph/9702430].

[13] N. Irges, S. Lavignac and P. Ramond, Predictions from an anomalous U(1) model of Yukawa hierarchies, Phys. Rev. D58 (1998) 035003, [hep-ph/ 9802334$].$ 
[14] J. K. Elwood, N. Irges and P. Ramond, Family symmetry and neutrino mixing, Phys. Rev. Lett. 81 (1998) 5064-5067, [hep-ph/9807228].

[15] L. Ferretti, S. F. King and A. Romanino, Flavour from accidental symmetries, JHEP 11 (2006) 078, [hep-ph/0609047].

[16] F. Simpson, R. Jimenez, C. Pena-Garay and L. Verde, Strong Bayesian Evidence for the Normal Neutrino Hierarchy, JCAP 1706 (2017) 029, [1703.03425].

[17] F. Capozzi, E. Di Valentino, E. Lisi, A. Marrone, A. Melchiorri and A. Palazzo, Global constraints on absolute neutrino masses and their ordering, Phys. Rev. D95 (2017) 096014, [1703. 04471 ].

[18] P. F. de Salas, D. V. Forero, C. A. Ternes, M. Tortola and J. W. F. Valle, Status of neutrino oscillations 2017, 1708.01186.

[19] S. Weinberg, Baryon and Lepton Nonconserving Processes, Phys. Rev. Lett. 43 (1979) 1566-1570.

[20] Y. Reyimuaji and A. Romanino, Can an unbroken flavour symmetry provide an approximate description of lepton masses and mixing?, JHEP 03 (2018) 067, [1801.10530].

[21] L. J. Hall, H. Murayama and N. Weiner, Neutrino mass anarchy, Phys. Rev. Lett. 84 (2000) 2572-2575, [hep-ph/9911341].

[22] N. Haba and H. Murayama, Anarchy and hierarchy, Phys. Rev. D63 (2001) 053010, [hep-ph/0009174].

[23] G. Altarelli and F. Feruglio, Tri-bimaximal neutrino mixing from discrete symmetry in extra dimensions, Nucl. Phys. B720 (2005) 64-88, [hep-ph/ 0504165$].$

[24] V. Domcke and A. Romanino, Stable lepton mass matrices, JHEP 06 (2016) 031, [160 4.08879 ].

[25] P. H. Frampton, S. T. Petcov and W. Rodejohann, On deviations from bimaximal neutrino mixing, Nucl. Phys. B687 (2004) 31-54, [hep-ph/ 0401206 ].

[26] A. Romanino, Charged lepton contributions to the solar neutrino mixing and $\theta_{13}$, Phys. Rev. D70 (2004) 013003, [hep-ph/0 402258$].$

[27] S. F. King, Predicting neutrino parameters from SO(3) family symmetry and quark-lepton unification, JHEP 08 (2005) 105, [hep-ph/ 0506297 ].

[28] S. Antusch and S. F. King, Charged lepton corrections to neutrino mixing angles and CP phases revisited, Phys. Lett. B631 (2005) 42-47, [hep-ph/ 0508044 ].

[29] K. A. Hochmuth, S. T. Petcov and W. Rodejohann, $U_{P M N S}=U_{l}^{\dagger} U_{v}$, Phys. Lett. B654 (2007) 177-188, [0706.2975].

[30] P. S. Bhupal Dev, R. N. Mohapatra and M. Severson, Neutrino Mixings in SO(10) with Type II Seesaw and $\theta_{13}$, Phys. Rev. D84 (2011) 053005, [1107.2378].

[31] S. Dev, S. Gupta and R. Raman Gautam, Parametrizing the Lepton Mixing Matrix in terms of Charged Lepton Corrections, Phys. Lett. B704 (2011) 527-533, [1107.1125].

[32] D. Marzocca, S. T. Petcov, A. Romanino and M. Spinrath, Sizeable $\theta_{13}$ from the Charged Lepton Sector in SU(5), (Tri-)Bimaximal Neutrino Mixing and Dirac CP Violation, JHEP 11 (2011) 009, [1108.0614].

[33] G. Altarelli, F. Feruglio and L. Merlo, Tri-Bimaximal Neutrino Mixing and Discrete Flavour Symmetries, Fortsch. Phys. 61 (2013) 507-534, [1205.5133]. 
[34] D. Marzocca, S. T. Petcov, A. Romanino and M. C. Sevilla, Nonzero $\left|U_{e 3}\right|$ from Charged Lepton Corrections and the Atmospheric Neutrino Mixing Angle, JHEP 05 (2013) 073, [1302. 0423 ].

[35] S. Gollu, K. N. Deepthi and R. Mohanta, Charged lepton correction to tribimaximal lepton mixing and its implications to neutrino phenomenology, Mod. Phys. Lett. A28 (2013) 1350131, [1303. 3393].

[36] D. Marzocca and A. Romanino, Stable fermion mass matrices and the charged lepton contribution to neutrino mixing, JHEP 11 (2014) 159, [1409.3760]. 